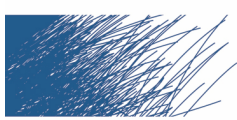

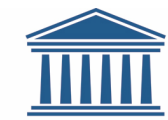 \\ UNIVERSITEIT \\ GENT
}

biblio.ugent.be

The UGent Institutional Repository is the electronic archiving and dissemination platform for all UGent research publications. Ghent University has implemented a mandate stipulating that all academic publications of UGent researchers should be deposited and archived in this repository. Except for items where current copyright restrictions apply, these papers are available in Open Access.

This item is the archived peer-reviewed author-version of:

Title: On the point behavior of Fourier and conjugate series

Authors: Ricardo Estrada and Jasson Vindas

In: Zeitschrift für Analysis und ihre Anwendungen 29 (4), 487-504, 2010

Optional: doi: $10.4171 /$ ZAA/1420

To refer to or to cite this work, please use the citation to the published version:

R. Estrada, J. Vindas (2010). On the point behavior of Fourier and conjugate series. Z. Anal. Anwend. 29 (4) 487-504. doi:10.4171/ZAA/1420 


\title{
ON THE POINT BEHAVIOR OF FOURIER SERIES AND CONJUGATE SERIES
}

\author{
RICARDO ESTRADA AND JASSON VINDAS
}

\begin{abstract}
We investigate the point behavior of periodic functions and Schwartz distributions when the Fourier series and the conjugate series are both Abel summable at a point. In particular we show that if $f$ is a bounded function and its Fourier series and conjugate series are Abel summable to values $\gamma$ and $\beta$ at the point $\theta_{0}$, respectively, then the primitive of $f$ is differentiable at $\theta_{0}$, with derivative equal to $\gamma$, the conjugate function satisfies $\lim _{\theta \rightarrow \theta_{0}}\left(3 /\left(\theta-\theta_{0}\right)^{3}\right) \int_{\theta_{0}}^{\theta} \tilde{f}(t)(\theta-t)^{2} \mathrm{~d} t=\beta$, and the Fourier series and the conjugate series are both $(\mathrm{C}, \kappa)$ summable at $\theta_{0}$, for any $\kappa>0$. We show a similar result for positive measures and $L^{1}$ functions bounded from below. Since the converse of our results are valid, we therefore provide a complete characterization of simultaneous Abel summability of the Fourier and conjugate series in terms of "average point values", within the classes of positive measures and functions bounded from below. For general $L^{1}$ functions, we also give a.e. distributional interpretation of $-(1 / 2 \pi)$ p.v. $\int_{-\pi}^{\pi} f\left(t+\theta_{0}\right) \cot (t / 2) \mathrm{d} t$ as the point value of the conjugate series when viewed as a distribution.

We obtain more general results of this kind for arbitrary trigonometric series with coefficients of slow growth, i.e., periodic distributions.
\end{abstract}

\section{INTRODUCTION}

It is well known that there is an intrinsic relationship between the local behavior of a function (or generalized function) at a point and the convergence or summability properties of its Fourier series and conjugate series [36]. Many classical results establish the summability of the series from the local behavior of the function $[7,8,17,18,32,33,35]$; some others go in the opposite direction, they obtain local information about the function itself from certain summability properties of the series [3, 10, 11, 12, 13, 16, 26]. The first problem is of Abelian nature while the second one usually has a Tauberian character [9]. Interestingly, sometimes, one can even go beyond the Abel-Tauber problem and provide precise characterizations relating a

2000 Mathematics Subject Classification. Primary 42A50, 42A24, 40E05. Secondary 40G05, 40G10, 46F10, 46F99.

Key words and phrases. Fourier series, conjugate series, Hilbert transform, pointwise behavior, distributions, Abel and Cesàro summability, distributional point values, Tauberian theorems, asymptotic behavior of generalized functions.

J. Vindas gratefully acknowledges support by a Postdoctoral Fellowship of the Research Foundation-Flanders (FWO, Belgium). 
summability method, or a family of them, with a specific type of point behavior $[3,10,11,12,13,26]$.

In this article we are concerned with problems related to Abel summability. The most basic result about Abel summability and Fourier series is due to Fatou $[7,36]$. Fatou's theorem states that if $f \in L^{1}[-\pi, \pi]$ with Fourier series

$$
\frac{a_{0}}{2}+\sum_{n=1}^{\infty} a_{n} \cos n \theta+b_{n} \sin n \theta,
$$

and its primitive is differentiable at the point $\theta=\theta_{0}$, i.e.,

$$
\lim _{\theta \rightarrow \theta_{0}} \frac{1}{\theta-\theta_{0}} \int_{\theta_{0}}^{\theta} f(t) \mathrm{d} t=\gamma
$$

then the Fourier series is Abel summable $[9,36]$ to the value $\gamma$ at $\theta=\theta_{0}$,

$$
\frac{a_{0}}{2}+\sum_{n=1}^{\infty} a_{n} \cos n \theta_{0}+b_{n} \sin n \theta_{0}=\gamma \quad(\mathrm{A}) \text {. }
$$

Loomis has shown two converses to Fatou theorem in [16]. He showed, using some results of Hardy and Littlewood [12,9], that if $f$ is a positive function and (3) holds, then the symmetric derivative of the primitive of $f$ exits and equals $\gamma$, i.e.,

$$
\lim _{\theta \rightarrow 0} \frac{1}{2 \theta} \int_{\theta_{0}-\theta}^{\theta_{0}+\theta} f(t) \mathrm{d} t=\gamma
$$

On the other hand (4) suffices to conclude (3). It should be observed that (2) always implies (4), but in general they are not equivalent. One may say that this result of Loomis is a Tauberian theorem, being the positivity of $f$ the Tauberian assumption; however, it is perhaps more appropriate to say that it is rather a characterization of Abel summability of Fourier series within the class of positive functions. Loomis also gave necessary and sufficient conditions to conclude (2) from (3) for positive functions, but this time an extra Tauberian hypothesis must be assumed. The results of Loomis apply to positive measures as well.

There is also a well known sort of version of Fatou theorem which gives Abel summability of the conjugate series [36]. One can show that if (2) is satisfied and the principal value integral,

$$
\beta=-\frac{1}{2 \pi} \mathrm{p} \cdot \mathrm{v} \cdot \int_{-\pi}^{\pi} f\left(t+\theta_{0}\right) \cot \left(\frac{t}{2}\right) \mathrm{d} t,
$$

exists, then the conjugate series is Abel summable as well,

$$
\sum_{n=1}^{\infty} a_{n} \sin n \theta_{0}-b_{n} \cos n \theta_{0}=\beta \quad(\mathrm{A}) .
$$


The scope of this article is to study the converse to this result, namely, we investigate point behavior when the Fourier series and the conjugate series are both simultaneously Abel summable at a point. We analyze the case of periodic functions, measures, and Schwartz distributions.

The plan of the article is as follows. Section 2 is of preliminary character, we discuss there the notion of average point values of functions and point values for distributions (in the sense of Eojasiewicz [15]), which are natural generalizations of (2). In Section 3 we study the existence of point values under the assumption of Abel summability of Fourier and conjugate series, our main result states that if either the distribution or the conjugate distribution (i.e., the one given by the conjugate series) is (distributionally) bounded at the point, then the point values of both distributions exist; the results of that section are essentially Tauberian theorems. We also give distributional interpretation of the conjugate integral (5) for $L^{1}$ functions as the a.e. distributional point value of the conjugate distribution. Section 4 deals with functions and measures, we provide characterizations of simultaneous Abel summability of the Fourier and conjugate series within the class of positive measures, in particular for bounded functions, in terms of (2) and an average point behavior of the conjugate distribution. We also obtain characterizations of this situation for functions and distributions which are bounded from below in a neighborhood of the analyzed point.

\section{Preliminaries AND Notation}

2.1. Notation. We denote by $\mathcal{D}^{\prime}(\mathbb{R})$ and $\mathcal{S}^{\prime}(\mathbb{R})$ the Schwartz spaces of distributions and tempered distributions. We refer to [21] for the very well known properties of these spaces, and to [5, 20, 23, 24, 31] for the theory of asymptotic expansions of distributions. We fix the constants in the Fourier transform so that

$$
\hat{\phi}(x)=\int_{-\infty}^{\infty} e^{-i x t} \phi(t) \mathrm{d} t, \text { for } \phi \in \mathcal{S}(\mathbb{R}) .
$$

We shall fix the complex exponential and cosines-sines Fourier expansions of a periodic function or distribution

$$
f(\theta)=\sum_{n=-\infty}^{\infty} c_{n} e^{i n \theta}=\frac{a_{0}}{2}+\sum_{n=1}^{\infty} a_{n} \cos n \theta+b_{n} \sin n \theta,
$$

and its conjugate distribution, namely, the distribution defined by

$$
\tilde{f}(\theta)=\sum_{n=-\infty}^{\infty} \tilde{c}_{n} e^{i n \theta}=\sum_{n=1}^{\infty} a_{n} \sin n \theta-b_{n} \cos n \theta .
$$

Observe that in general $\tilde{f}$ is not a function but a distribution, even if $f$ is a function; indeed there exists $f \in L^{1}[-\pi, \pi]$ such that $\tilde{f}$ is not integrable over any finite interval [36, Chap.VII, p.257]. 
We will consider limits in the Cesàro sense $[9,5]$. Given $g$, a locally integrable function, we write

$$
\lim _{x \rightarrow \infty} g(x)=\ell \quad(\mathrm{C}, k)
$$

if

$$
\lim _{x \rightarrow \infty} \frac{k}{x} \int_{0}^{x} g(t)\left(1-\frac{t}{x}\right)^{k-1} \mathrm{~d} t=\ell .
$$

If we do not want to make reference to $k$, we simply write $(\mathrm{C})$ for $(\mathrm{C}, k)$.

2.2. Average Point Values of Functions. Let $f$ be a locally integrable function. Except when the function is continuous, it does not make much sense to speak about an individual point value as one does for "usual functions". Indeed, one usually regards two functions which are equal a.e. as the same object, so changing a function in a set of measure zero, for instance a point, does not change the object we call "function" in analysis. Therefore, the concept of point value is rather an average notion; for example, one may use Lebesgue points or relation (2) as the actual point values of the function $f$.

It is natural to consider generalizations of (2) and take higher order averages, such an idea goes back to Hardy and Littlewood [11], and also to Łojasiewicz [15]. Let $k \in \mathbb{N}$. In analogy with (8), we shall say that $f$ has a point value in the $(\mathrm{C}, k)$ sense and write $f\left(\theta_{0}\right)=\gamma(\mathrm{C}, k)$ if

$$
\lim _{\theta \rightarrow \theta_{0}} \frac{k}{\left(\theta-\theta_{0}\right)^{k}} \int_{\theta_{0}}^{\theta} f(t)(\theta-t)^{k-1} \mathrm{~d} t=\gamma .
$$

We may also say that $f$ has an average point value of order $k$ at $\theta=\theta_{0}$. Observe that (9) also makes sense for a (regular) Borel measure $\mu$, one simply has to replace $f(t) \mathrm{d} t$ by $\mathrm{d} \mu(t)$. In this case we also write $\mu\left(\theta_{0}\right)=\gamma \quad(\mathrm{C}, k)$. It is easy to see that (9) implies that $\mu$ is continuous at $\theta=\theta_{0}$, i.e., the measure of $\left\{\theta_{0}\right\}$ with respect to $\mu$ is zero, $\mu\left(\left\{\theta_{0}\right\}\right)=0$.

2.3. Distributional Point Values and Fourier Series. The notion of point values for Schwartz distributions was introduced by Łojasiewicz in [15]. A distribution $f \in \mathcal{D}^{\prime}(\mathbb{R})$ is said to have a distributional point value at the point $\theta=\theta_{0}$ if there exist a non-negative integer $k$ and a function $F$, locally integrable in some neighborhood of $\theta_{0}$, such that $F^{(k)}=f$ near $\theta_{0}$ and the following limit exists

$$
\lim _{\theta \rightarrow \theta_{0}} \frac{k ! F(\theta)}{\left(\theta-\theta_{0}\right)^{k}}=\gamma .
$$

In such a case we say that $\gamma$ is the value of $f$ at $\theta=\theta_{0}$ and write $f\left(\theta_{0}\right)=\gamma$, distributionally. If (10) holds we say that the point value is of order $k$. Notice that if the distribution is locally integrable near the point, then this definition is exactly the same as (9), but in general a distribution can have distributional point values without being locally integrable [15]. 
There is an useful equivalent definition of distributional point values, which is actually Łojasiewicz original definition. It can be shown $[15,31]$ that $f\left(\theta_{0}\right)=\gamma$, distributionally, if and only if the following limit exists in the weak topology of $\mathcal{D}^{\prime}(\mathbb{R})$,

$$
\lim _{\varepsilon \rightarrow 0} f\left(\theta_{0}+\varepsilon \theta\right)=\gamma, \quad \text { in } \mathcal{D}^{\prime}(\mathbb{R}),
$$

namely, for each test function $\phi \in \mathcal{D}(\mathbb{R})$

$$
\lim _{\varepsilon \rightarrow 0} \frac{1}{\varepsilon}\left\langle f(\theta), \phi\left(\frac{\theta-\theta_{0}}{\varepsilon}\right)\right\rangle=\gamma \int_{-\infty}^{\infty} \phi(\theta) \mathrm{d} \theta .
$$

If $f \in \mathcal{S}^{\prime}(\mathbb{R})$, then (11) also holds for each $\phi \in \mathcal{S}(\mathbb{R})[4,31]$.

When $f$ is a $2 \pi$-periodic distribution, it is possible to characterize [3] its point values in terms of a certain summability of the Fourier series (6). Indeed, $f\left(\theta_{0}\right)=\gamma$, distributionally, if and only if there exists a non-negative integer $m$ such that

$$
\text { e.v. } \sum_{n=-\infty}^{\infty} c_{n} e^{i n \theta_{0}}=\gamma \quad(\mathrm{C}, m),
$$

where (12) means that all the following slightly asymmetric means converge

$$
\lim _{x \rightarrow \infty} \sum_{-x<n \leq a x} c_{n} e^{i n \theta_{0}}=\gamma \quad(\mathrm{C}, m), \text { for each } a>0 .
$$

Remarkably, an analog result is true for Fourier transforms [26, 30]. We also refer to $[4,6,24,25,27,29,32,33]$ for further results in this direction.

It is important to point out that (12) is not equivalent to the Cesàro summability of the two series $\sum_{n=-\infty}^{1} c_{n} e^{i n \theta_{0}}$ and $\sum_{n=0}^{\infty} c_{n} e^{i n \theta_{0}}$, separately; counterexamples can be found in $[24,26]$. On the other hand, if these two series are Cesàro summable for some $m$, then it is easy to see that the distributional point values of $f$ and $\tilde{f}$ must exist at $\theta=\theta_{0}$, and the converse assertion also holds. We must also emphasize a notational aspect, we will use the symbol $\tilde{f}\left(\theta_{0}\right)$ to denote the distributional point value of the distribution given by (7), we will show, in Corollary 3.2 below, that when $f$ is locally integrable it agrees almost everywhere with the usual conjugate function [36] given by the principal value integral (5).

We shall need two more local concepts for distributions, those of distributional boundedness at a point [34] and jump behavior [6, 29]. We say that a distribution is distributionally bounded at $\theta=\theta_{0}$ if there exist $k \in \mathbb{N}$ and a $k$-primitive $F$ of $f$ which is locally integrable in a neighborhood of $\theta_{0}$ and $F(\theta)=O\left(\left(\theta-\theta_{0}\right)^{k}\right), \theta \rightarrow \theta_{0}$. We say that $f$ has a jump behavior if $k !\left(\theta-\theta_{0}\right)^{-k} F(\theta) \rightarrow \gamma_{ \pm}$, as $\theta \rightarrow \theta_{0}^{ \pm}$; in this case we write $f\left(\theta_{0}^{ \pm}\right)=\gamma_{ \pm}$, distributionally, its distributional right and left point values; the jump of $f$ is then defined as the number $[f]_{\theta=\theta_{0}}=\gamma_{+}-\gamma_{-}=f\left(\theta_{0}^{+}\right)-f\left(\theta_{0}^{-}\right)$. Distributional boundedness is equivalent to the weak boundedness of $f\left(\theta_{0}+\varepsilon \theta\right)$ as $\varepsilon \rightarrow 0$, i.e., for each test function $\left\langle f\left(\theta_{0}+\varepsilon \theta\right), \phi(\theta)\right\rangle=O(1)$. 


\section{Point Behavior of Distributions and Abel Summability of Fourier and Conjugate Series}

We discuss in this section results which relate the Abel summability of the Fourier series and conjugate series with the local point behavior of a distribution and its conjugate distribution. Our first theorem does this when the distribution has a jump behavior. The meaning of $(\mathrm{C})$ below is $(\mathrm{C}, k)$ for some $k$.

Theorem 3.1. Let $f$ be a $2 \pi$-periodic distribution with Fourier series (6). Suppose that $f$ has jump behavior at $\theta=\theta_{0}$. If

$$
\sum_{n=1}^{\infty}\left(a_{n} \sin n \theta_{0}-b_{n} \cos n \theta_{0}\right)=\beta \quad(\mathrm{A})
$$

then $f$ and $\tilde{f}$ have Eojasiewicz point value at $\theta=\theta_{0}$, that is, $f\left(\theta_{0}\right)=f\left(\theta_{0}^{ \pm}\right)$, and $\tilde{f}\left(\theta_{0}\right)=\beta$, distributionally. Moreover,

$$
\sum_{n=0}^{\infty} c_{n} e^{i n \theta_{0}}=\frac{f\left(\theta_{0}\right)+i \tilde{f}\left(\theta_{0}\right)}{2}+\frac{c_{0}}{2} \quad(\mathrm{C})
$$

and

$$
\sum_{n=-\infty}^{1} c_{n} e^{i n \theta_{0}}=\frac{f\left(\theta_{0}\right)-i \tilde{f}\left(\theta_{0}\right)}{2}-\frac{c_{0}}{2} \quad(\mathrm{C})
$$

Proof. The distributional version of Lukács theorem $[4,29]$ (see the classical one in $[17,19,36])$ and the Abel summability of the conjugate series imply that

$$
\beta+o(1)=\sum_{n=1}^{\infty}\left(a_{n} \sin n \theta_{0}-b_{n} \cos n \theta_{0}\right) r^{n} \sim \frac{[f]_{\theta=\theta_{0}}}{\pi} \log (1-r),
$$

as $r \rightarrow 1^{-}$, and so $[f]_{\theta=\theta_{0}}=0$; therefore $f\left(\theta_{0}\right)=f\left(\theta_{0}^{ \pm}\right)$, distributionally. Next, the results from $[26,23]$ imply the existence of a continuous function $c$ such that the following two distributional asymptotics hold

$$
\sum_{n=0}^{\infty} c_{n} e^{i n \theta_{0}} \delta(\lambda x-n) \sim\left(\frac{f\left(\theta_{0}\right)}{2}+c(\lambda)\right) \frac{\delta(x)}{\lambda}, \quad \text { as } \lambda \rightarrow \infty,
$$

and

$$
\sum_{n=-\infty}^{1} c_{n} e^{i n \theta_{0}} \delta(\lambda x-n) \sim\left(\frac{f\left(\theta_{0}\right)}{2}-c(\lambda)\right) \frac{\delta(x)}{\lambda}, \quad \text { as } \lambda \rightarrow \infty,
$$

where $\delta$ is the Dirac delta distribution and both (15) and (16) are interpreted in the weak topology of $\mathcal{S}^{\prime}(\mathbb{R})$. Multiplying $(15)$ and (16) by $-i \operatorname{sgn} n$, evaluating (15) at $e^{-x}$ and (16) at $e^{x}$, and adding the two results, we get, as 


$$
\begin{aligned}
\lambda \rightarrow \infty, & \\
\beta+o(1) & =\sum_{n=1}^{\infty}\left(a_{n} \sin n \theta_{0}-b_{n} \cos n \theta_{0}\right) e^{-\frac{n}{\lambda}} \\
& =i\left(\frac{f\left(\theta_{0}\right)}{2}-c(\lambda)\right)\left\langle\delta(x), e^{x}\right\rangle-i\left(\frac{f\left(\theta_{0}\right)}{2}+c(\lambda)-c_{0}\right)\left\langle\delta(x), e^{-x}\right\rangle \\
& =-2 i c(\lambda)+i c_{0},
\end{aligned}
$$

and so

$$
c(\lambda)=\frac{c_{0}+i \beta}{2}+o(1), \quad \lambda \rightarrow \infty .
$$

But (15)-(17) together give us

$$
\sum_{n=-\infty}^{\infty} \tilde{c}_{n} e^{i n \theta_{0}} \delta(\lambda x-n) \sim \beta \frac{\delta(x)}{\lambda}, \quad \text { as } \lambda \rightarrow \infty \text { in } \mathcal{S}^{\prime}(\mathbb{R}),
$$

and, by [26, Lem.1], the last fact is equivalent to the existence of the point value of $\tilde{f}$ at $\theta=\theta_{0}$, namely, $\tilde{f}\left(\theta_{0}\right)=\beta$, distributionally. On the other hand, inserting (17) into (15) and (16) and using [26, Cor.7], we obtain at once (13) and (14).

Theorem 3.1 allows us to give distributional interpretation to the conjugate function of an $L^{1}$ function. Indeed, recall that if $f \in L^{1}[-\pi, \pi]$, at every point $\theta_{0}$ where the derivative of the primitive of $f$ exists [36, Thm.7.20, Chap.III],

$\sum_{n=1}^{\infty}\left(a_{n} \sin n \theta_{0}-b_{n} \cos n \theta_{0}\right) r^{n}+\frac{1}{2 \pi} \int_{1-r}^{\pi}\left(f\left(t+\theta_{0}\right)-f\left(\theta_{0}-t\right)\right) \cot \left(\frac{t}{2}\right) \mathrm{d} t \rightarrow 0$,

as $r \rightarrow 1^{-}$. So, at those points, the Abel summability of the conjugate series is equivalent to the existence of the principal value integral. It is also very well known that the conjugate series is Abel summable almost everywhere [36, Chap.VII, p.252]. Since the Łojasiewicz point value exists at any point where the primitive has a derivative, we immediately obtain from Theorem 3.1 the following corollary.

Corollary 3.2. Let $f \in L^{1}[-\pi, \pi]$, then $\tilde{f}$ has Eojasiewicz point value at every point $\theta_{0}$ where the derivative of the primitive of $f$ exists and the conjugate series is Abel summable. Moreover, at those points,

$$
\tilde{f}\left(\theta_{0}\right)=-\frac{1}{2 \pi} \text { p.v. } \int_{-\pi}^{\pi} f\left(t+\theta_{0}\right) \cot \left(\frac{t}{2}\right) \mathrm{d} t, \quad \text { distributionally. }
$$

Furthermore, the equality (18) holds almost everywhere.

We may obtain a stronger result than Theorem 3.1, but we have to pay the price of passing through a Tauberian theorem [28, Thm.3.2]. The next theorem is the most important of this section. 
Theorem 3.3. Let $f$ be a $2 \pi$-periodic distribution with Fourier series (6). Suppose that

$$
\frac{a_{0}}{2}+\sum_{n=1}^{\infty}\left(a_{n} \cos n \theta_{0}+b_{n} \sin n \theta_{0}\right)=\gamma \quad(\mathrm{A})
$$

and

$$
\sum_{n=1}^{\infty}\left(a_{n} \sin n \theta_{0}-b_{n} \cos n \theta_{0}\right)=\beta \quad(\mathrm{A}) .
$$

If either $f$ or $\tilde{f}$ is distributionally bounded at $\theta=\theta_{0}$, then $f\left(\theta_{0}\right)=\gamma$ and $\tilde{f}\left(\theta_{0}\right)=\beta$, distributionally. Furthermore (13) and (14) hold.

Proof. By symmetry, we may assume that $f$ is distributionally bounded at $\theta=\theta_{0}$. Let $U$ and $V$ be the standard harmonic representations [2,33] of $f$ and $\tilde{f}$ on the upper half-plane $\Im m z>0$, that is,

$$
U(z)=\sum_{n=0}^{\infty} c_{n} e^{i n z}+\sum_{n=-\infty}^{1} c_{n} e^{i n \bar{z}} \text { and } V(z)=\sum_{n=1}^{\infty} \tilde{c}_{n} e^{i n z}+\sum_{n=-\infty}^{1} \tilde{c}_{n} e^{i n \bar{z}}
$$

Put $G(z)=U(z)+i V(z)=-c_{0}+2 \sum_{n=0}^{\infty} c_{n} e^{i n z}$. Then $G$ is analytic in the upper half-plane $\Im m z>0$, it has a radial limit at $\theta=\theta_{0}$, i.e., $\lim _{y \rightarrow 0^{+}} G\left(\theta_{0}+i y\right)=\gamma+i \beta$, and it has distributional boundary values on the real axis, actually the boundary distribution is obviously $g(\theta)=$ $-c_{0}+2 \sum_{n=0}^{\infty} c_{n} e^{i n \theta}=f(\theta)+i \tilde{f}(\theta)$.

Suppose that we were able to show that $g$ is distributionally bounded at $\theta=\theta_{0}$, since $G$ has a radial value, then the hypotheses of the Tauberian theorem from [28, Thm.3.2] would be fully satisfied and it would lead to the conclusion $g\left(\theta_{0}\right)=\gamma+i \beta$, distributionally, which implies that $f\left(\theta_{0}\right)=$ $\gamma$ and $\tilde{f}\left(\theta_{0}\right)=\beta$, distributionally; moreover, the latter would obviously yield (13) and (14), since the distributional point values of the distributions $\sum_{n=0}^{\infty} c_{n} e^{i n \theta}$ and $\sum_{n=-\infty}^{1} c_{n} e^{i n \theta}$ would be $\left(\gamma+i \beta+c_{0}\right) / 2$ and $\left(\gamma-i \beta-c_{0}\right) / 2$, respectively. So the proof of the present theorem will be complete after we establish the distributional boundedness of $g$ at $\theta=\theta_{0}$.

Let us show the distributional boundedness of $g$ at $\theta=\theta_{0}$. Observe that the distributional boundedness of $f$ explicitly means that $f\left(\theta_{0}+\varepsilon \theta\right)=O(1)$, $\varepsilon \rightarrow 0^{+}$, in the weak topology of $\mathcal{S}^{\prime}(\mathbb{R})$, so by applying the Fourier transform, we obtain that, as $\lambda \rightarrow \infty$,

$$
\sum_{n=-\infty}^{\infty} c_{n} e^{i n \theta_{0}} \delta(\lambda x-n)=O\left(\frac{1}{\lambda}\right), \quad \text { in } \mathcal{S}^{\prime}(\mathbb{R}) .
$$

The results from $[23,25]$ imply that there exists a continuous function $c(\lambda)$ such that

$$
\sum_{n=1}^{\infty} c_{n} e^{i n \theta_{0}} \delta(\lambda x-n)=c(\lambda) \frac{\delta(x)}{\lambda}+O\left(\frac{1}{\lambda}\right), \quad \text { in } \mathcal{S}^{\prime}(\mathbb{R})
$$


and

$$
\sum_{n=-\infty}^{1} c_{n} e^{i n \theta_{0}} \delta(\lambda x-n)=-c(\lambda) \frac{\delta(x)}{\lambda}+O\left(\frac{1}{\lambda}\right), \quad \text { in } \mathcal{S}^{\prime}(\mathbb{R}) .
$$

The same argument used in the proof of Theorem 3.1 and the hypothesis (20) yield $c(\lambda)=O(1), \lambda \rightarrow \infty$, and hence

$$
-c_{0} \delta(\lambda x)+2 \sum_{n=0}^{\infty} c_{n} e^{i n \theta_{0}} \delta(\lambda x-n)=O\left(\frac{1}{\lambda}\right), \quad \text { in } \mathcal{S}^{\prime}(\mathbb{R}),
$$

taking inverse Fourier transform in the above relation, we convince ourselves that $g$ is distributionally bounded at $\theta=\theta_{0}$. This completes the proof.

Remark 3.4. Theorem 3.3 includes Theorem 3.1. Indeed, the existence of the jump behavior gives in particular distributional boundedness at the point, it also implies the Abel summability of the Fourier series [4, 25].

There is also another useful notion of point value which is weaker than the one of Łojasiewicz, that of symmetric point value $[5,24,30]$. It is related to de la Vallée Poussin generalized symmetric derivatives [36, Chap.XI]. One says that a distribution $f$ has a symmetric (distributional) point value $\gamma$ at $\theta=\theta_{0}$, if its symmetric part about $\theta=\theta_{0}$,

$$
\chi(\theta)=\frac{f\left(\theta+\theta_{0}\right)+f\left(\theta-\theta_{0}\right)}{2},
$$

has $\gamma$ as point value at $\theta=0$, that is, $\chi(0)=\gamma$, distributionally. In such a case one writes $f_{\text {sym }}\left(\theta_{0}\right)=\gamma$, distributionally. Naturally, the existence of the distributional point value implies the existence of the symmetric point value, but the converse is not true, as shown by the example $\delta^{\prime}(\theta)$ at $\theta=0$; indeed, $\delta_{\text {sym }}^{\prime}(0)=0$, distributionally, because its symmetric part about the origin vanishes, but obviously the value of $\delta^{\prime}$ does not exist at $\theta=0$. Observe that (4) is a particular case of this distributional concept. The existence of the symmetric point value at $\theta=\theta_{0}$ implies the Abel summability of the Fourier series at the point $[5,24,30]$. Thus, we obtain from Theorem 3.3 the following corollary.

Corollary 3.5. Let $f$ be a $2 \pi$-periodic distribution with Fourier series (6). Suppose that $f_{\mathrm{sym}}\left(\theta_{0}\right)=\gamma$, distributionally. If (20) holds and if either $f$ or $\tilde{f}$ is distributionally bounded at $\theta=\theta_{0}$, then $f\left(\theta_{0}\right)=\gamma$ and $\tilde{f}\left(\theta_{0}\right)=\beta$, distributionally. Furthermore (13) and (14) hold.

\section{Point Behavior of Periodic Functions and Measures}

In this section we provide complete characterizations of Abel summability at a point of the Fourier and conjugate series for functions and positive measures in terms of average point values. More generally, we extend the characterization to distribution which are bounded from below in a neighborhood of the analyzed point. 
4.1. Bounded Functions. We now combine Theorem 3.3 with some results of Hardy and Littlewood $[10,11,12,13]$ to obtain the ensuing theorem about functions which are locally bounded at a point.

Theorem 4.1. Let $f \in L^{1}[-\pi, \pi]$ be bounded in a neighborhood of the point $\theta=\theta_{0}$. Let (6) be its Fourier series. Then, we have simultaneously the Abel summability of its Fourier series and conjugate series (19) and (20) if and only if $f\left(\theta_{0}\right)=\gamma \quad(\mathrm{C}, 1)$ and $\tilde{f}\left(\theta_{0}\right)=\beta \quad(\mathrm{C}, 3)$. Namely, the first order primitive of $f$ is differentiable at $\theta=\theta_{0}$,

$$
\lim _{\theta \rightarrow \theta_{0}} \frac{1}{\theta-\theta_{0}} \int_{\theta_{0}}^{\theta} f(t) \mathrm{d} t=\gamma
$$

and the conjugate distribution (which is integrable near $\theta=\theta_{0}$ ) satisfies

$$
\lim _{\theta \rightarrow \theta_{0}} \frac{3}{\left(\theta-\theta_{0}\right)^{3}} \int_{\theta_{0}}^{\theta} \tilde{f}(t)(\theta-t)^{2} \mathrm{~d} t=\beta .
$$

Furthermore, under these circumstances,

$$
\beta=-\frac{1}{2 \pi} \text { p.v. } \int_{-\pi}^{\pi} f\left(t+\theta_{0}\right) \cot \left(\frac{t}{2}\right) \mathrm{d} t .
$$

In addition, we have $(\mathrm{C}, \kappa)$ summability of the series for any $\kappa>0$,

$$
\sum_{n=0}^{\infty} c_{n} e^{i n \theta_{0}}=\frac{\gamma+i \beta+c_{0}}{2} \quad(\mathrm{C}, \kappa),
$$

and

$$
\sum_{n=-\infty}^{1} c_{n} e^{i n \theta_{0}}=\frac{\gamma-i \beta-c_{0}}{2} \quad(\mathrm{C}, \kappa) .
$$

Proof. The converse is clear since the existence of the two point values implies Abel summability [4, 24, 25, 33].

Observe that the local boundedness of $f$ gives us for free the distributional boundedness of $f$ at $\theta=\theta_{0}$. So, Theorem 3.3 yields at once $f\left(\theta_{0}\right)=\gamma$, distributionally, but since $f$ is a bounded function, then the point value must be of order 1 [15] and then (21) follows. We have already seen that under these circumstances, (21) and (20), the principal value integral in (23) exists and agrees with $\beta$. A theorem of Hardy and Littlewood [10] (see also $[11,12])$ gives us that if $(21)$ holds, then

$$
\frac{a_{0}}{2}+\sum_{n=1}^{\infty} a_{n} \cos n \theta_{0}+b_{n} \sin n \theta_{0}=\gamma \quad(\mathrm{C}, \kappa),
$$

for any $\kappa>0$. Theorem 3.3 also implies $\tilde{f}\left(\theta_{0}\right)=\beta$, distributionally, but not the assertion about the order of the point value. It should be noticed that $\tilde{f}$ is integrable in a neighborhood of $\theta=\theta_{0}$, it is implied by the local boundedness of $f$. On the other hand $\tilde{f}\left(\theta_{0}\right)=\beta$, distributionally, implies 
the Cesàro summability of the conjugate series [3, 26], but another theorem of Hardy and Littlewood [13] implies

$$
\sum_{n=1}^{\infty} a_{n} \sin n \theta_{0}-b_{n} \cos n \theta_{0}=\beta \quad(\mathrm{C}, \kappa),
$$

for any $\kappa>0$.

Using $c_{n}=\left(a_{|n|}-i \operatorname{sgn} n b_{|n|}\right) / 2$, it is easy to see that (24) and (25) follow from (26) and (27). Finally, invoking the equivalence theorem between Riesz and Cesàro means $[9,14],(24)$ and $(25)$ give

$$
\text { e.v. } \sum_{n=-\infty}^{\infty} \tilde{c}_{n} e^{i n \theta_{0}}=\beta \quad(\mathrm{C}, 1)
$$

and this fact in combination with [30, Thm.5.2] yield $\tilde{f}\left(\theta_{0}\right)=\beta \quad(\mathrm{C}, 3)$, which in turn is equivalent to (22).

In particular, we obtain the characterization of simultaneous Abel summability of the Fourier and conjugate series for bounded functions.

Corollary 4.2. Let $f \in L^{\infty}[-\pi, \pi]$ have Fourier series (6). Then, simultaneous Abel summability of its Fourier series and conjugate series (19) and (20) is equivalent to (21) and (22).

4.2. Positive Measures and Functions Bounded from Below. Theorem 4.1 does not tell all the true, it is also valid for $L^{1}$ functions which are bounded from below. A version for positive measures also holds. We need the following lemma in order to establish those facts. In the following, we shall write $\int_{a}^{b}$ for integration over the closed interval $[a, b]$, if we write $\int_{a^{+}}^{b}$ it means integration over $(a, b]$, a similar meaning is assigned to the symbols $\int_{a}^{b^{-}}$and $\int_{a^{+}}^{b^{-}}$. Given a $2 \pi$-periodic measure $\mu$, we use the following definition for its Fourier coefficients

$$
a_{n}=\frac{1}{\pi} \int_{-\pi^{+}}^{\pi} \cos n t \mathrm{~d} \mu(t) \quad \text { and } \quad b_{n}=\frac{1}{\pi} \int_{-\pi^{+}}^{\pi} \sin n t \mathrm{~d} \mu(t),
$$

so that the Fourier expansion converges to $\mu$ in the space $\mathcal{S}^{\prime}(\mathbb{R})$. The integral $\int_{-\pi}^{\pi^{-}}$could have also be used and we would obtain the same result. On the other hand if we use $\int_{-\pi}^{\pi}$ then the Fourier expansion does not converge to $\mu$ in general, it rather converges to the periodic distribution $\mu+\mu(\{\pi\}) \sum_{n=-\infty}^{\infty} \delta(\cdot-(2 n+1) \pi)$. After these preliminaries, we state the lemma.

Lemma 4.3. Let $\mu$ be a $2 \pi$-periodic positive measure with Fourier series (6). If its Abel-Poisson means are bounded at the point $\theta=\theta_{0}$, that is,

$$
\frac{a_{0}}{2}+\sum_{n=1}^{\infty}\left(a_{n} \cos n \theta_{0}+b_{n} \sin n \theta_{0}\right) r^{n}=O(1), \quad r \rightarrow 1^{-},
$$


then $\mu$ is distributionally bounded at $\theta=\theta_{0}$. Moreover, $\mu$ is continuous at $\theta=\theta_{0}$, i.e., $\mu\left(\left\{\theta_{0}\right\}\right)=0$, and

$$
\frac{1}{\left(\theta-\theta_{0}\right)} \int_{\theta_{0}}^{\theta} \mathrm{d} \mu(t)=O(1), \quad \theta \rightarrow \theta_{0} .
$$

Proof. We may assume that $\theta_{0}=0$, by translating. Observe next that (28) says

$$
\int_{-\pi^{+}}^{\pi} P_{r}(t) \mathrm{d} \mu(t)=O(1), \quad r \rightarrow 1^{-},
$$

where $P_{r}$ is the Poisson kernel, i.e., $P_{r}(\theta)=\left(1-r^{2}\right) /\left(1-2 r \cos \theta+r^{2}\right)$. Define the following primitive of $\mu$

$$
F(\theta)= \begin{cases}\int_{0}^{\theta} \mathrm{d} \mu(\theta) & \theta>0, \\ \int_{0^{-}}^{\theta} \mathrm{d} \mu(\theta), & \theta \leq 0,\end{cases}
$$

Thus, $F$ is non-decreasing, right continuous on $(0, \infty)$, and left continuous on $(-\infty, 0]$ with $F(0)=F\left(0^{-}\right)=0$. Let us show that $\mu$ is continuous at the origin, that is, $F\left(0^{+}\right)=0$. From (29), we have that

$$
0 \leq \int_{-\pi^{+}}^{0^{-}} P_{r}(t) \mathrm{d} \mu(t)+\int_{0^{+}}^{\pi} P_{r}(t) \mathrm{d} \mu(t)+F\left(0^{+}\right) \frac{1+r}{1-r}=O(1), \quad r \rightarrow 1^{-},
$$

but the three terms in this inequality are positive, so each of them must be bounded, and hence $F\left(0^{+}\right)=0$. Working with the second integral, integrating by parts, and using that $-P_{r}^{\prime}(\theta)$ is positive for $\theta>0$, we have,

$$
\begin{aligned}
O(1) & =\int_{0}^{\pi} P_{r}(t) \mathrm{d} \mu(t)=F(\pi) P_{r}(\pi)+\int_{0}^{\pi}\left(-P_{r}^{\prime}(t)\right) F(t) \mathrm{d} t \\
& \geq \int_{1-r}^{\pi}\left(-P_{r}^{\prime}(t)\right) F(t) \mathrm{d} t \geq F(1-r) \int_{1-r}^{\pi}\left(-P_{r}^{\prime}(t)\right) \mathrm{d} t \\
& =\frac{F(1-r)}{1-r}\left(\frac{1+r}{1+\frac{2 r-2 r \cos (1-r)}{(1-r)^{2}}}-\frac{(1-r)^{2}}{1+r}\right) \\
& \sim \frac{F(1-r)}{(1-r)}, \quad r \rightarrow 1^{-} .
\end{aligned}
$$

Therefore,

$$
\frac{F(\theta)}{\theta}=O(1), \quad \theta \rightarrow 0^{+} .
$$

The integral over $(-\pi, 0)$ can be handled in a similar manner to obtain the estimate $\theta^{-1} F(\theta)=O(1), \theta \rightarrow 0^{-}$. Thus, the proof is complete.

We then obtain from Theorem 3.3 and Lemma 4.3 the following result for periodic positive measures. 
Theorem 4.4. Let $\mu$ be a $2 \pi$-periodic positive measure with Fourier series (6). Then, we have simultaneously the Abel summability of its Fourier series and conjugate series (19) and (20) if and only if $\mu\left(\theta_{0}\right)=\gamma \quad(\mathrm{C}, 1)$, namely its first order primitive is differentiable at $\theta=\theta_{0}$,

$$
\lim _{\theta \rightarrow \theta_{0}} \frac{1}{\theta-\theta_{0}} \int_{\theta_{0}}^{\theta} \mathrm{d} \mu(t)=\gamma
$$

and the conjugate distribution $\tilde{\mu}$ has a point value of order 3 at $\theta=\theta_{0}$, $\tilde{\mu}\left(\theta_{0}\right)=\beta$, distributionally. In addition,

$$
\beta=-\frac{1}{2 \pi} \text { p.v. } \int_{-\pi^{+}}^{\pi} \cot \left(\frac{t-\theta_{0}}{2}\right) \mathrm{d} \mu(t) .
$$

and we have $(\mathrm{C}, \kappa)$ summability of the series for any $\kappa>0$,

$$
\sum_{n=0}^{\infty} c_{n} e^{i n \theta_{0}}=\frac{\gamma+i \beta+c_{0}}{2} \quad(\mathrm{C}, \kappa),
$$

and

$$
\sum_{n=-\infty}^{1} c_{n} e^{i n \theta_{0}}=\frac{\gamma-i \beta-c_{0}}{2} \quad(\mathrm{C}, \kappa) .
$$

Proof. Lemma 4.3 gives the distributional boundedness of $\mu$ at $\theta=\theta_{0}$. So, Theorem 3.3 yields $\mu\left(\theta_{0}\right)=\gamma$, distributionally. For positive measures the existence of the distributional point value is equivalent to (30), as shown by Łojasiewicz in [15]. Theorem 3.3 also implies $\tilde{\mu}\left(\theta_{0}\right)=\beta$, distributionally. Now the theorem of Young-Riesz-Plessner [36, Thm.8.1, Chap.III] implies (31), under the assumption of Abel summability of the conjugate series (one has to integrate by parts and use [36, Thm.7.15, Chap.III]). The rest of the proof is identically the same as the one of Theorem 4.1, but using [36, Thm.8.1, Chap.III] instead of the results from [10, 13].

We now provide the announced extension of Theorem 4.1 to functions bounded from below (or above).

Theorem 4.5. Let $f \in L^{1}[-\pi, \pi]$ be bounded from below (or above) in some neighborhood of $\theta=\theta_{0}$. Let (6) be its Fourier series. The simultaneous Abel summability of the Fourier series and conjugate series (19) and (20) is equivalent to $f\left(\theta_{0}\right)=\gamma \quad(\mathrm{C}, 1)$, namely,

$$
\lim _{\theta \rightarrow \theta_{0}} \frac{1}{\theta-\theta_{0}} \int_{\theta_{0}}^{\theta} f(t) \mathrm{d} t=\gamma
$$

and the existence of the point value of order 3 at $\theta=\theta_{0}$ for the conjugate distribution, $\tilde{f}\left(\theta_{0}\right)=\beta$, distributionally. Furthermore,

$$
\beta=-\frac{1}{2 \pi} \text { p.v. } \int_{-\pi}^{\pi} f\left(t+\theta_{0}\right) \cot \left(\frac{t}{2}\right) \mathrm{d} t,
$$

and we have $(\mathrm{C}, \kappa)$ summability of the series (32) and (33) for any $\kappa>0$. 
Proof. The statement of the present theorem is a local one, we can therefore assume the existence of a constant $K>0$ such that $f>-K$, globally. Apply now Theorem 4.4 to the positive measure $\mu=f+K$.

Remark 4.6. It has been pointed out in Section 2 that (32) and (33) are sufficient to establish the existence of the point values of $f$ and $\tilde{f}$. Therefore, for positive measures and functions bounded from below (or above) in a neighborhood of the point, they are necessary and sufficient for the simultaneous Abel summability of the Fourier and conjugate series.

4.3. Distributions Bounded from Below (Above). The global integrability of $f$ on $[-\pi, \pi]$ can be removed from Theorem 4.5 in order to allow more general trigonometric series in the statement.

Given $f \in \mathcal{D}^{\prime}(\mathbb{R})$ and a point $\theta_{0} \in \mathbb{R}$, we shall say that $f$ is bounded from below (above) in a neighborhood of $\theta=\theta_{0}$ if there exist $\sigma, K \geq 0$ such that $-K \leq f$ on $\left(\theta_{0}-\sigma, \theta_{0}+\sigma\right)$ (resp. $\left.f \leq K\right)$, i.e., for each non-negative $\phi \in \mathcal{D}\left(\theta_{0}-\sigma, \theta_{0}+\sigma\right)$

$$
-K \int_{-\infty}^{\infty} \phi(\theta) \mathrm{d} \theta \leq\langle f(\theta), \phi(\theta)\rangle \quad\left(\text { resp. } K \int_{-\infty}^{\infty} \phi(\theta) \mathrm{d} \theta \geq\right) .
$$

It should be noticed that under this circumstances $f$ is a signed measure in a neighborhood of the point.

We end this article by characterizing simultaneous Abel summability of the Fourier and conjugate series for distributions which satisfy (34) near the point. We first show a lemma.

Lemma 4.7. Let $g$ be a $2 \pi$-periodic distribution vanishing in a neighborhood of the point $\theta=\theta_{0}$. Then the conjugate distribution $\tilde{f}$ is a $C^{\infty}$-function in a neighborhood of $\theta=\theta_{0}$, and consequently the Fourier series and conjugate series of $g$ are Abel summable to the values 0 and $\tilde{g}\left(\theta_{0}\right)$, respectively.

Proof. Let $U$ and $V$ be the standard harmonic representations of $f$ and $\tilde{f}$ on $\Im m z>0$, i.e., the ones used in the proof of Theorem 3.3. By applying the reflection principle to the real and imaginary parts of $U$ ([1, Sec.4.5], [22, Sec.3.4]), we have that $U$ admits a harmonic extension to a (complex) neighborhood of $\theta_{0}$. Since $V$ is harmonic conjugate to $U$, we conclude that $V$ admits a harmonic extension to a (complex) neighborhood of $\theta_{0}$ as well. Therefore, $\tilde{f}$ is a $C^{\infty}$-function near $\theta=\theta_{0}$. The Abel summability of the two series is implied by the results from $[4,25]$.

Theorem 4.8. Let the $2 \pi$-periodic distribution $f \in \mathcal{D}^{\prime}(\mathbb{R})$ be bounded from below (or above) in some neighborhood of $\theta=\theta_{0}$. Let (6) be its Fourier series. The following properties are equivalent:

(i) The simultaneous Abel summability of the Fourier series and conjugate series (19) and (20).

(ii) The existence of the point values $f\left(\theta_{0}\right)=\gamma \quad(\mathrm{C}, 1)$ and $\tilde{f}\left(\theta_{0}\right)=\beta$, distributionally of order 3. 
(iii) The existence of the distributional point values of $f$ and $\tilde{f}$ at $\theta=\theta_{0}$, $f\left(\theta_{0}\right)=\gamma$ and $\tilde{f}\left(\theta_{0}\right)=\beta$, distributionally.

(iv) The existence of some (possibly large) $\kappa$ such that

$$
\frac{a_{0}}{2}+\sum_{n=1}^{\infty}\left(a_{n} \cos n \theta_{0}+b_{n} \sin n \theta_{0}\right)=\gamma \quad(\mathrm{C}, \kappa),
$$

and

$$
\sum_{n=1}^{\infty}\left(a_{n} \sin n \theta_{0}-b_{n} \cos n \theta_{0}\right)=\beta \quad(\mathrm{C}, \kappa) .
$$

(v) The existence of some (possibly large) $\kappa$ such that

$$
\sum_{n=0}^{\infty} c_{n} e^{i n \theta_{0}}=\frac{\gamma+i \beta+c_{0}}{2} \quad(\mathrm{C}, \kappa)
$$

and

$$
\sum_{n=-\infty}^{1} c_{n} e^{i n \theta_{0}}=\frac{\gamma-i \beta-c_{0}}{2} \quad(\mathrm{C}, \kappa) .
$$

Proof. Observe that (iii), (iv) and (v) are equivalent in general, regardless the assumption over $f$; moreover, clearly, any of them implies (i) [4, 25].

It remains to show that (i) implies (ii) under the hypothesis $f$ is bounded from below in a neighborhood of $\theta=\theta_{0}$. By adding a constant $K>0$, we can assume that $f$ is a positive measure in a neighborhood of $\theta=\theta_{0}$. So, we can decompose $f=g+\mu$, where $g$ is a $2 \pi$-periodic distribution which vanishes in a neighborhood of $\theta=\theta_{0}$, and $\mu$ is a $2 \pi$-periodic positive measure. By Lemma 4.7 and the assumption (i), we have that the Fourier and conjugate series of $\mu$ are both Abel summable at $\theta=\theta_{0}$, (ii) now follows directly from Theorem 4.4 and Lemma 4.7.

\section{REFERENCES}

[1] E. J. Beltrami, M. R. Wohlers, Distributions and the Boundary Values of Analytic Functions, Academic Press, N.Y., 1966.

[2] H. Bremermann, Distributions, Complex Variables and Fourier Transforms, AddisonWesley Publishing Co., Massachusetts-London, 1965.

[3] R. Estrada, Characterization of the Fourier series of a distribution having a value at a point, Proc. Amer. Math. Soc. 124 (1996), 1205-1212.

[4] R. Estrada, A distributional version of the Ferenc Lukács theorem, Sarajevo J. Math. 1 (2005), 75-92.

[5] R. Estrada, R. P. Kanwal, A distributional approach to asymptotics. Theory and applications, Second edition, Birkhäuser, Boston, 2002.

[6] R. Estrada, J. Vindas, Determination of jumps of distributions by differentiated means, Acta Math. Hungar. 124 (2009), 215-241.

[7] P. Fatou, Séries trigonométriques et séries de Taylor, Acta Math. 30 (1906), 335-400.

[8] L. Fejér, Über die Bestimmung des Sprunges der Funktion aus ihrer Fourierreihe, J. Reine Angew. Math. 142 (1913), 165-188.

[9] G. H. Hardy, Divergent Series, Clarendon Press, Oxford, 1949.

[10] G. H. Hardy, J. E. Littlewood, On the Fourier series of a bounded function, Proc. London Math. Soc. 17 (1918), xiii-xiv (Records of Proceedings at Meetings). 
[11] G. H. Hardy, J. E. Littlewood, Solution of the Cesàro summability problem for power series and Fourier series, Math. Z. 19 (1923), 67-96.

[12] G. H. Hardy, J. E. Littlewood, Notes on the theory of series-II: The Fourier series of a positive function, J. London Math. Soc. 1 (1926), 134-138.

[13] G. H. Hardy, J. E. Littlewood, On series conjugate to Fourier series of bounded function, J. London Math. Soc. 6 (1931), 278-281.

[14] A. E. Ingham, The equivalence theorem for Cesàro and Riesz summability, Publ. Ramanujan Inst. 1 (1968), 107-113.

[15] S. Łojasiewicz, Sur la valuer et la limite d'une distribution en un point, Studia Math. 16 (1957), 1-36.

[16] L. H. Loomis, The converse of the Fatou theorem for positive harmonic functions, Trans. Amer. Math. Soc. 53 (1943), 239-250.

[17] F. Lukács, Über die Bestimmung des Sprunges einer Funktion aus ihrer Fourierreihe, J. Reine Angew. Math. 150 (1920), 107-112.

[18] M. L. Misra, On the summability of the conjugate series of a Fourier series by logarithmic means, Proc. Nat. Inst. Sci. India 13 (1947), 157-168.

[19] F. Móricz, Ferenc Lukács type theorems in terms of the Abel-Poisson means of conjugate series, Proc. Amer. Math. Soc. 131 (2003), 1243-1250.

[20] S. Pilipović, B. Stanković, A. Takači, Asymptotic behaviour and Stieltjes transformation of distributions, Teubner-Texte zur Mathematik, Leipzig, 1990.

[21] L. Schwartz, Théorie des Distributions, Hermann, Paris, 1966.

[22] E. C. Titchmarsh, The Theory of Functions, Second edition, Oxford University Press, Oxford, 1939.

[23] J. Vindas, Structural theorems for quasiasymptotics of distributions at infinity, Publ. Inst. Math. (Beograd) (N.S.) 84(98) (2008), 159-174.

[24] J. Vindas, Local Behavior of Distributions and Applications, Dissertation, Louisiana State University, 2009.

[25] J. Vindas, The structure of quasiasymptotics of Schwartz distributions, Linear and non-linear theory of generalized functions and its applications, pp. 297-314, Banach Center Publ. 88, Polish Acad. Sc. Inst. Math., Warsaw, 2010.

[26] J. Vindas, R. Estrada, Distributional Point Values and Convergence of Fourier Series and Integrals, J. Fourier. Anal. Appl. 13 (2007), 551-576.

[27] J. Vindas, R. Estrada, Distributionally Regulated Functions, Studia Math. 181 (2007), 211-236.

[28] J. Vindas, R. Estrada, A tauberian theorem for distributional point values, Arch. Math. (Basel) 91 (2008), 247-253.

[29] J. Vindas, R. Estrada, On the jump behavior of distributions and logarithmic averages, J. Math. Anal. Appl. 347 (2008), 597-606.

[30] J. Vindas, R. Estrada, On the order of summability of the Fourier inversion formula, Anal. Theory Appl. 26 (2010), 13-42.

[31] J. Vindas, S. Pilipović, Structural theorems for quasiasymptotics of distributions at the origin, Math. Nachr. 282 (2009), 1584-1599.

[32] G. Walter, Pointwise convergence of distribution expansions, Studia Math. 26 (1966), 143-154.

[33] G. Walter, Fourier series and analytic representation of distributions, SIAM Review 12 (1970), 272-276.

[34] Z. Zielézny, Über die Mengen der regulären und singulären Punkte einer Distribution, Studia Math. 19 (1960), 27-52.

[35] A. Zygmund, Sur un théorème de M. Gronwall, Bull. Acad. Polon. (1925), 207-217.

[36] A. Zygmund, Trigonometric Series, Vols. I, II, Second edition, Cambridge University Press, Cambridge, 1959. 
Department of Mathematics, Louisiana State University, Baton Rouge, LA 70803-4918, U.S.A.

E-mail address: restrada@math.1su.edu

Department of Mathematics, Ghent University, Krijgslaan 281 Gebouw S22, B 9000 Gent, Belgium

E-mail address: jvindas@cage.Ugent.be 